\title{
Spotlight on cervical vagus nerve stimulation for the treatment of primary headache disorders: a review
}

This article was published in the following Dove Press journal:

Journal of Pain Research

\author{
Ilana S Lendvai ${ }^{1,2}$ \\ Ayline Maier ${ }^{1,2}$ \\ Dirk Scheele ${ }^{1,2}$ \\ Rene Hurlemann ${ }^{1,2}$ \\ Thomas M Kinfe ${ }^{1,2}$ \\ 'Department of Psychiatry, Rheinische \\ Friedrich-Wilhelms University, \\ Bonn, Germany; ${ }^{2}$ Department of \\ Psychiatry and Medical Psychology, \\ University Hospital Bonn, Rheinische \\ Friedrich-Wilhelms University, Bonn, \\ Germany
}

Objectives: Cervical noninvasive vagus nerve stimulation (nVNS) emerged as an adjunctive neuromodulation approach for primary headache disorders with limited responsiveness to pharmacologic and behavioral treatment. This narrative review evaluates the safety and efficacy of invasive and noninvasive peripheral nerve stimulation of the cervical branch of the vagal nerve (afferent properties) for primary headache disorders (episodic/chronic migraine [EM/ $\mathrm{CM}]$ and cluster headache $[\mathrm{ECH} / \mathrm{CCH}]$ ) and provides a brief summary of the preclinical data on the possible mechanism of action of cervical vagus nerve stimulation (VNS) and trigeminonociceptive head pain transmission.

Materials and methods: A systematic search of published data was performed in PubMed for randomized controlled trials (RCTs) and prospective cohort clinical studies assessing the efficacy/safety and cost-effectiveness of cervical VNS in primary headache disorders and related preclinical studies.

Results: Three RCTs were identified for ECH/CCH (ACT-1, ACT-2 and PREVA), one RCT for migraine (EVENT) and several prospective cohort studies and retrospective analyses for both headache disorders. In ACT-1, a significantly higher response rate, a higher pain-free rate and a decrease in mean attack duration were found in nVNS-treated $\mathrm{ECH} / \mathrm{CCH}$ patients compared to sham stimulation. ACT-2 confirmed these findings (e.g., significantly higher pain-free attacks, pain severity decline and increased responder-rate [defined as $\geq 50 \%$ reduction]). The PREVA study demonstrated the superiority of adjunctive nVNS to standard care alone and observed a significantly higher attack reduction $(p=0.02)$ and responder rate (defined as $\geq 50 \%$ reduction). For CM, the EVENT study assessed a significantly higher frequency of decline in the open-label phase. Mostly transient mild/moderate adverse events were recorded, and no severe device-related adverse events occurred.

Conclusion: Cervical nVNS represents a novel, safe and efficient adjunctive treatment option for primary headache disorders. In particular, preliminary observations suggest enhanced nVNS responsiveness in favor of episodic subtypes (EM and ECH). However, preclinical studies are urgently warranted to dissect the mechanism of action.

Keywords: cervical vagus nerve stimulation, migraine, cluster headache, safety/efficacy, trigemino-nociceptive signaling, neuroinflammation

\section{Introduction}

Department of Psychiatry and Medical Psychology, University Hospital Bonn, Rheinische Friedrich-Wilhelms University, Sigmund-Freud Street 25, 53I27 Bonn, Germany

Tel +49228287 19123

Fax +4922828716772

Email thomas.kinfe@ukb.uni-bonn.de
In the past two decades, surgically implanted cervical vagal nerve stimulation (iVNS; Cyberonics Inc., TX, USA) was investigated in clinical trials in a broad variety of neurologic disorders such as epilepsy, depression and Alzheimer's disease. ${ }^{1-3}$

Chronic daily headache $(\mathrm{CDH})$ and/or migrane occur particularly frequent in an underestimated proportion of seizure patients. ${ }^{4}$ The impact of iVNS on 
seizure-associated head pain was first reported $>10$ years ago. ${ }^{5-7}$ However, only two pilot studies have been specifically conducted for the treatment of primary headache disorders. Including migraine and cluster headache, both pilot studies demonstrated encouraging efficacy in terms of $50 \%$ reduction in severity/frequency with adjunctive iVNS ${ }^{8,9}$ More recently, a retrospective, large database analysis found a sustained, clinically meaningful impact of iVNS in seizure-related CDH and migraine. ${ }^{10}$ In spite of this marked observed effect, iVNS requires surgical implantation and has been associated with a considerable percentage of implantation- and/or stimulationassociated side effects, diminishing the otherwise beneficial VNS outcome. ${ }^{3}$ In view of the currently available abortive pharmacologic interventions (e.g., triptans) with pain-free response rates in migraine of $30 \%$ at $2 \mathrm{~h}$ and $20 \%$ at $24 \mathrm{~h}$, and in cluster headache of $45 \%$ at $15 \mathrm{~min}$ after rescue drug intake, novel adjunctive acute and preventive treatment alternatives are urgently needed to counterbalance the long-term pharmacologic side effects and/or limited responsiveness. ${ }^{11}$

Thus, a cervical non-invasive, equally effective approach has been developed (noninvasive vagus nerve stimulation [nVNS]; gammaCore, NJ, USA), and the capability to perform preventive and abortive neuromodulation therapy for migraine and cluster headache with a distinct lower incidence of adverse events (AEs) has been demonstrated. ${ }^{11-29}$ Cervical nVNS represents a portable neuromodulation device that received CE mark approval for the acute and preventive treatment of primary headache disorders (migraine, cluster headache) and medication-overuse headache, and was approved by the US Food and Drug Administration for the acute treatment of episodic cluster headache and acute pain associated with migraine.

The scope of this article is to provide a narrative review of randomized controlled trials (RCTs), prospective cohort studies, retrospective analyses and cost-effectiveness assessments in order to determine the impact, safety and tolerability of cervical $\mathrm{nVNS}$ as an adjunctive treatment of primary headache disorders with a focus on episodic and chronic migraine (EM/CM) and cluster headache (ECH/CCH). Specified parameters were the following: cervical iVNS (invasive; surgically implanted), cervical nVNS (noninvasive, transcutaneous), primary headache disorders (migraine and cluster headache), severity and frequency, prevention and acute head pain treatment, study year/design, observation period, stimulation paradigm, the safety and tolerability profile of VNS, trigeminal allodynia, trigemino-nociceptive system, trigemino-cervical (vascular) complex, neuroinflammation, experimental head pain model and cortical spreading depression (CSD).

In addition, this review briefly covers experimental studies to highlight the postulated mechanism of action (MOA) of VNS in head pain models with emphasis on the neuroinflammation genesis of primary headache disorders and possible interactions with trigemino-nociceptive headache signaling.

Other VNS neuromodulation approaches targeting, for instance, the auricular branch of the vagal nerve were per definition not part of this review, as well as preliminary reports of otherwise unpublished data.

\section{General characteristics and class of evidence of cervical VNS studies}

Between 2000 and 2018, two clinical trials (pilot studies) were published using iVNS specifically addressed to migraine and cluster headache patients. As yet, there exist mainly case reports and/or retrospective assessments of iVNS seizure patients with coincidental $\mathrm{CDH}$, migraine or other forms of headache. Mainly due to the low number of patients investigated and the study design, evidence-based conclusion about iVNS head pain outcome is limited (Table 1). ${ }^{5-10}$

Specifically targeting EM and CM, one RCT (EVENT: Chronic migraine prevention with non-invasive vagus nerve stimulation; Class II study) and five prospective observational, cohort studies have been extracted for the years 2014-2018 (data from PRESTO study not included). All included trials were conducted to determine, in particular, the preventive and abortive impact of adjunctive, cervical nVNS. In some instances, patients were included and classified as not drug resistant (Table 2). ${ }^{4,11-18}$

Clinical trials conducted to assess nNVS for ECH or $\mathrm{CCH}$ (e.g., preventive and abortive capability) exist in higher numbers and on a higher level of evidence. Three RCTs (ACT-1: Non-invasive vagus nerve stimulation for the acute treatment of cluster headache; ACT-2: Non-invasive vagus nerve stimulation for the acute treatment of episodic and chronic cluster headache, PREVA: Non-invasive vagus nerve stimulation for prevention and acute treatment of chronic cluster headache) and two prospective case series were published in the past 3 years (2015-2018). ${ }^{23,25-29}$ Distinct study designs were conceptualized in order to separately screen the acute (ACT-1, ACT-2) and the preventive (PREVA) outcome of $\mathrm{ECH} / \mathrm{CCH}$ patients treated with cervical nVNS. . $5,26,28,29$ Additionally, a post hoc analysis and a cost-effectiveness evaluation were performed according to the PREVA data (Table 3). 
Table I Summary of clinical studies addressed to invasive cervical vagal nerve stimulation (iVNS) and primary headache disorders

\begin{tabular}{|c|c|c|c|c|c|c|c|c|}
\hline $\begin{array}{l}\text { Year, study } \\
\text { design }\end{array}$ & $\begin{array}{l}\text { Headache } \\
\text { disorder }\end{array}$ & $\begin{array}{l}\text { Primary } \\
\text { indication }\end{array}$ & $\begin{array}{l}\text { Patient } \\
\text { no. }\end{array}$ & $\begin{array}{l}\text { Outcomel } \\
\text { parameter }\end{array}$ & Follow-up & $\begin{array}{l}\text { Cyclic } \\
\text { stimulation } \\
\text { paradigm }\end{array}$ & Efficacy & $\begin{array}{l}\text { Safetyl } \\
\text { tolerability }\end{array}$ \\
\hline $\begin{array}{l}2000, \text { rCS }^{5} \\
\text { Class IV }\end{array}$ & CM & Seizure & 1 & Severity/frequency & 10 years & $\begin{array}{l}30 \mathrm{sec} \text { on } / \mathrm{I}-5 \\
\mathrm{~min} \text { off } \\
20 \mathrm{~Hz}, 200-250 \\
\mu \mathrm{sec}\end{array}$ & $\mathrm{R}$ & Not reported \\
\hline $\begin{array}{l}\text { 2002, pCS } \\
\text { Class IV }\end{array}$ & CM & Seizure & 1 & Severity/frequency & 2 months & Not reported & $\mathrm{R}$ & Not reported \\
\hline $\begin{array}{l}2003, \mathrm{rCS}^{7} \\
\text { Class IV }\end{array}$ & CM & Seizure & 4 & Severity/frequency & 14 years & $\begin{array}{l}30 \mathrm{sec} \text { on/l-5 } \\
\mathrm{min} \text { off } \\
20 \mathrm{~Hz}, 200-250 \\
\mu \mathrm{sec}\end{array}$ & R-75\% & Not reported \\
\hline $\begin{array}{l}\text { 2005, pPS } \\
\text { Class IV }\end{array}$ & $\begin{array}{l}\mathrm{CM} / \mathrm{CCH} / \\
\mathrm{BM}\end{array}$ & Headache & $\begin{array}{l}6 \\
3 \mathrm{CM} \\
2 \mathrm{CCH} \\
1 \mathrm{BM}\end{array}$ & $\begin{array}{l}\text { Severity/frequency } \\
\text { Functional } \\
\text { impairment } \\
\text { (MIDAS) }\end{array}$ & 6 months & Not reported & $\begin{array}{l}\text { R-66\% (2 CM }) \\
\text { R- } 100 \%(2 \mathrm{CCH})\end{array}$ & $\begin{array}{l}\text { Vomitus BM } \\
\text { patient }\end{array}$ \\
\hline $\begin{array}{l}\text { 2009, pPS } \\
\text { Class IV }\end{array}$ & CM & $\begin{array}{l}\text { Headache } \\
\text { Depressive } \\
\text { disorder }\end{array}$ & 4 & $\begin{array}{l}\text { Severity/frequency } \\
\text { Functional } \\
\text { impairment } \\
\text { (MIDAS/HRSD) }\end{array}$ & 4-14 months & $\begin{array}{l}30 \mathrm{sec} \text { on } / \mathrm{I}-5 \\
\mathrm{~min} \text { off } \\
\mathrm{I} .25-2.5 \mathrm{~mA} \text {, } \\
30 \mathrm{~Hz} \\
500 \mu \mathrm{sec}\end{array}$ & R- $100 \%$ & $\begin{array}{l}\text { Voice alteration, } \\
\text { dyspnea, cough }\end{array}$ \\
\hline $\begin{array}{l}\text { 2017, } \mathrm{rCS}^{10} \\
\text { Class IV }\end{array}$ & CM & $\begin{array}{l}\text { Seizure } \\
\text { iVNS+SoC } \\
\text { vs. SoC vs. } \\
\text { HC }\end{array}$ & $\begin{array}{l}19 \\
10 \text { iVNS } \\
9 \text { SoC }\end{array}$ & $\begin{array}{l}\text { Severity/frequency } \\
\text { Affective/cognitive } \\
\text { head pain } \\
\text { perception } \\
\text { (MIDAS, PASS-40, } \\
\text { FSVA) }\end{array}$ & $5-13$ years & $\begin{array}{l}30 \mathrm{sec} \text { on/ } /-5 \\
\min \text { off } \\
0.5-2 \mathrm{~mA}, 20 \mathrm{~Hz} \text {, } \\
200-250 \mu \mathrm{sec}\end{array}$ & $\begin{array}{l}\text { iVNS (VAS) } 5.4 \\
\text { vs. SoC (VAS) } \\
7.8, p=0.03 \\
\text { iVNS (PASS) } 21 \\
\text { vs. SoC (PASS) } \\
16, p=0.02\end{array}$ & $\begin{array}{l}\text { Voice disturbance } \\
\text { Battery } \\
\text { replacement }\end{array}$ \\
\hline
\end{tabular}

Abbreviations: $\mathrm{BM}$, basilar migraine; $\mathrm{CCH}$, chronic cluster headache; $\mathrm{CH}$, cluster headache; $\mathrm{CM}$, chronic migraine; FSVA, questionnaire for pain-associated vigilance and attention; HC, healthy control; HRSD, Hamilton Rating Scale for Depression; iVNS, surgically implanted cervical vagal nerve stimulation; MIDAS, Migraine Disability Scale; PASS, Pain Anxiety Symptoms Scale; pCS, prospective case series; pPS, prospective pilot study; R, responder ( $\geq 30 \%-50 \%$ reduction severity/frequency); rCS, retrospective case series; SoC, standard of care; VAS, visual analog scale.

\section{Cervical invasive (surgically implanted) VNS and primary headache disorders}

The majority of the published iVNS data consist of case series or retrospective analyses, predominantly in patients with refractory focal seizures with co-occurring chronic headache (commonly migraine) with follow-up varying from 3 months to 14 years. ${ }^{3,5-10}$ For surgically implanted VNS, a multicenter, randomized controlled study has not yet been carried out, as seizure mostly represented the primary indication. Thus, a sufficient and comparative analysis of stimulation patterns and surgical- and/or stimulation-induced side effects addressed to head pain outcome is limited.

The majority of the 26 patients (basilar migraine, $\mathrm{CCH}$, $\mathrm{CDH}, \mathrm{CM}$ ) were treated by iVNS., ${ }^{3,5-7}$ Two pilot studies dedicated to refractory headache in 10 patients have been published. ${ }^{8,9}$ The first included six patients (three CM, two $\mathrm{CCH}$ and one basilar migraine). Two of three patients with $\mathrm{CM}$ and both $\mathrm{CCH}$ patients improved markedly with respect to severity and functional impairment. The second pilot trial enrolled four patients (solely CM) and observed a $100 \%$ responder rate, as all four patients $(\mathrm{CM})$ responded (defined as at least $50 \%$ reduction in severity or frequency).

The largest previous study was a retrospective analysis including 325 seizure patients ( $6 \%$ with coincidental CDH or CM [19/325]) that compared iVNS/standard medical care (SMC) vs. SMC alone vs. an age-/gender-matched healthy control (HC) group with an observation period ranging from 5 to 13 years (mean 8 years). ${ }^{10}$

iVNS was applied utilizing the following cyclic stimulation paradigms: $1.3 \mathrm{~mA}(0.5-2 \mathrm{~mA}), 20 \mathrm{~Hz}, 250 \mu \mathrm{s}, 30 \mathrm{sec}$ on/1.9 min off ( $0.5-5 \mathrm{~min})$. iVNS outcome parameters were headache severity/frequency and functional capacity (mood changes, sleep, cognitive pain perception, pain-associated anxiety and fear behavior). The iVNS group experienced a significantly lower headache severity (visual analog scale [VAS] scores [iVNS 5.4, SMC 7.8; $p=0.03$ ]) accompanied by functional responsiveness measured by the Pain Anxiety 


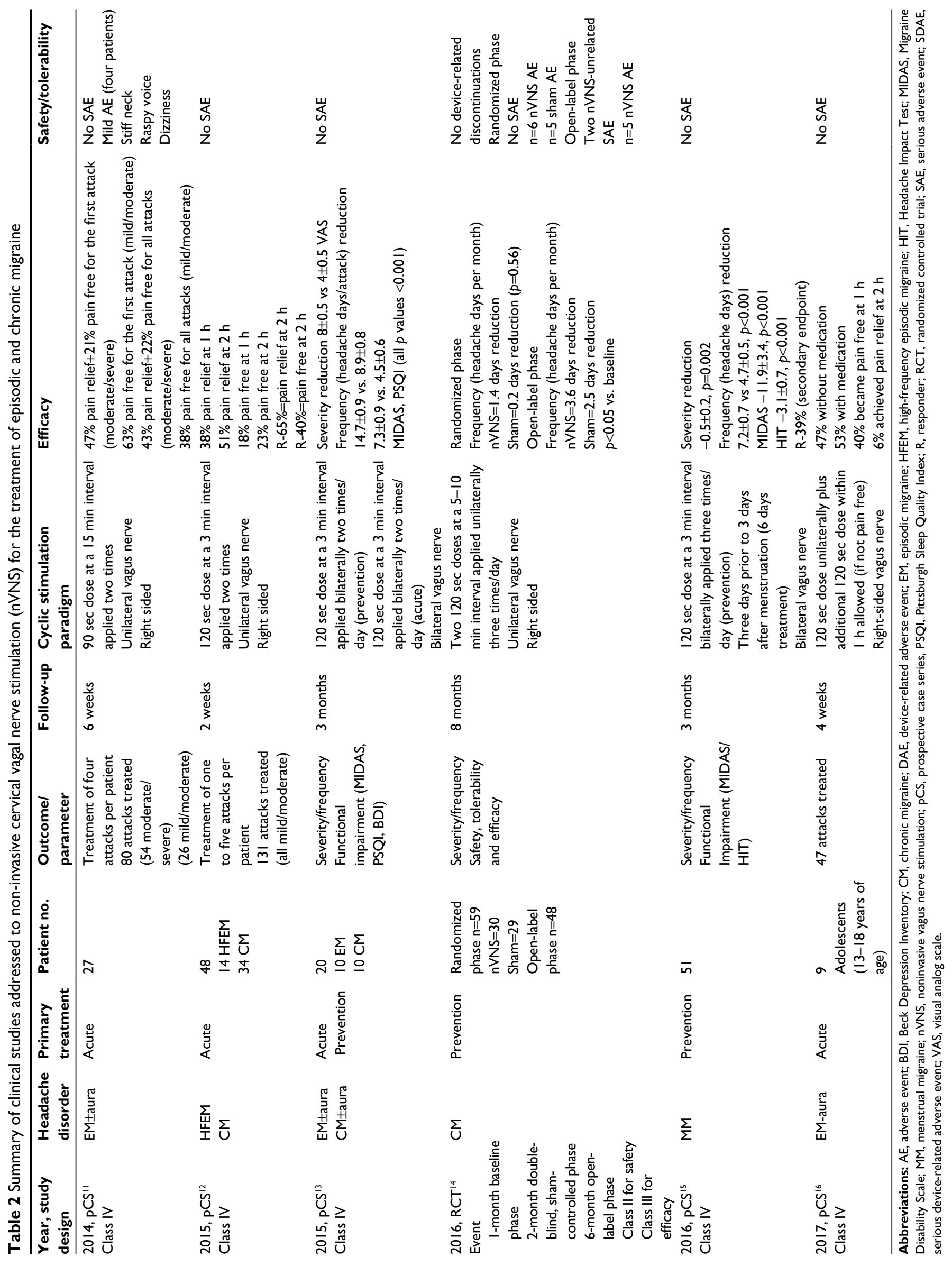


Symptom Score (cognitive/anxiety subscores [iVNS 21, SMC 16 ; $p=0.02]$ ) compared to SMC and HC. Other functional state parameters were not significantly different (Table 1).

However, the earlier data (Class IV studies) suggest that iVNS attenuates seizure-associated head pain and primary headache disorders. Although not determined on a high evidence level, in some instances, reduction of head pain severity/frequency was superior to seizure improvement.

\section{Cervical noninvasive VNS and primary headache disorders Episodic and chronic migraine}

Several prospective cohort studies have been published assessing the preventive and acute usefulness of cervical nVNS for EM and CM and migraine-associated comorbidities. ${ }^{11-14}$ In addition migraine subtypes such as menstrualrelated migraine and migraine in young adolescents have been under clinical investigation. ${ }^{15-17}$

Goadsby et al first introduced nVNS as an adjunctive alternative for abortive treatment in EM with/without aura. ${ }^{11}$ In an open-label study design, cervical nVNS was assessed for its adjunctive and abortive impact in $27 \mathrm{EM}$ patients (19 with moderate-severe head pain and 8 with mild-moderate head pain), who in total treated 80 attacks. nVNS was unilaterally (right sided) applied with a $90 \mathrm{sec}$ dose at a $15 \mathrm{~min}$ interval. Participants were permitted to treat up to four attacks (Table 2). Adjunctive nVNS for the first treated attack demonstrated a pain-free rate, at $2 \mathrm{~h}$ after treatment, of $21 \%(4 / 19)$ and a pain relief rate of $47 \%(9 / 19)$ in the moderate-severe classified group. Also, 63\% (5/8) in the mild-moderate rated group experienced a pain-free state. For all mild-moderate attacks, 38\% (10/26) achieved freedom from pain, and for all attacks classified as moderate-severe, 22\% (12/54) were pain free at $2 \mathrm{~h}$ and $43 \%(23 / 54)$ achieved pain relief. Thus, the abortive effect of cervical nVNS for mild-moderate attacks as well as for moderate-severe classified head pain was comparably effective with first-line pharmacologic interventions. These initial observations were extended and confirmed toward EM and CM and migraine-related impaired functional capacity such as mood changes and sleep quality. ${ }^{12,13}$ In the first clinical trial, both migraine subtypes (EM and $\mathrm{CM}$ ) were evaluated.

Adjunctive cervical nVNS was applied abortively over 2 weeks with an acute treatment protocol ( $120 \mathrm{sec}$ dose, unilateral, right sided, at $3 \mathrm{~min}$ intervals, two times/day) and achieved a pain relief rate of $38 \%$ at $1 \mathrm{~h}$ and $51 \%$ at $2 \mathrm{~h}$ after treatment and a pain-free rate of $18 \%$ at $1 \mathrm{~h}$ and $23 \%$ at $2 \mathrm{~h}$. Out of the $48 \mathrm{EM} / \mathrm{CM}$ patients, $56 \%$ reported pain relief at $1 \mathrm{~h}$ and $65 \%$ at $2 \mathrm{~h}^{12}$ The second EM/CM cohort study extended the use of $n V N S$ toward prevention and abortive use and found a significant decline of severity ( $8 \pm 0.5$ vs $4 \pm 0.5$ VAS, $p<0.001)$ and frequency (headache days: $14.7 \pm 0.9$ vs. $8.9 \pm 0.8$, attacks: 7.30 .9 vs. $4.5 \pm 0.6 ; p<0.001)$ and improved functional capacity (e.g., sleep quality, mood, and migraine disability, $p<0.001)$ after 3 months adjunctive $n$ VNS (prevention protocol: $120 \mathrm{sec}$ dose, bilateral, at $3 \mathrm{~min}$ interval; two times/day plus acute protocol: $120 \mathrm{sec}$ dose, bilateral, at $3 \mathrm{~min}$ interval two times for abortive use). ${ }^{13}$ Both studies found nVNS to be effective in EM compared to chronic migraine and that nVNS may improve head pain-related disability. Notably and contrary to the pilot study of Goadsby et al, the nVNS paradigm was slightly modified as nVNS was applied at a $120 \mathrm{sec}$ dose for a shorter interval.

A randomized double-blind, sham-controlled multicenter trial (EVENT) solely evaluated the preventive value and safety/tolerability in chronic migraine with a slightly different stimulation protocol compared to the previous migraine/ nVNS studies. ${ }^{14}$ In total, $48 \mathrm{CM}$ patients were enrolled and a meaningful frequency reduction was observed in the randomized phase (nVNS -1.4 days vs. 0.2 days), while the open-label phase nVNS (120 sec dose, right sided, at 5-10 min interval) was associated with significantly decreased headache days/month ( $\mathrm{nVNS}-3.6$ vs. -2.5 days, $p<0.05$ ). Of note, the EVENT study failed to achieve its primary endpoint, as a $50 \%$ reduction was only confirmed in $39 \%$ of nVNStreated subjects. Most of the observed AEs (treatment related or device related) were of mild character and transient. No device-associated discontinuation and/or device-associated severe AEs were recorded. ${ }^{14}$

Furthermore, menstrual-related migraine and migraine in young adolescents have been under nVNS investigation, as both migraine subtypes are limited either in preventive/ abortive responsiveness to conventional pharmacotherapy or in the availability of conventional interventions in the case of young migraine patients. ${ }^{15,16}$ The first study assessed 51 menstrual/menstrual-associated migraine patients treated with 3 months adjunctive, prophylactic nVNS (prevention protocol: $120 \mathrm{sec}$ dose, bilateral, at $3 \mathrm{~min}$ interval, three times/day, nVNS initiation 3 days prior to 3 days after menstruation onset). Thirty-nine percent (20/51) perceived a $\geq 50 \%$ reduction with a significant decline in frequency (headache days/ month: $7.2 \pm 0.5$ vs $4.7 \pm 0.5, p<0.001$ ), severity (VAS reduction: $-0.5 \pm 0.2, p=0.002$ ) and functional responsiveness (migraine disability score: $-11.9 \pm 0.5$, headache impact test: $-3.1 \pm 0.7 ; p<0.001) .{ }^{15}$

The second study included nine adolescent migraineurs (EM; age: 13-18 years), who treated 47 attacks in total within 


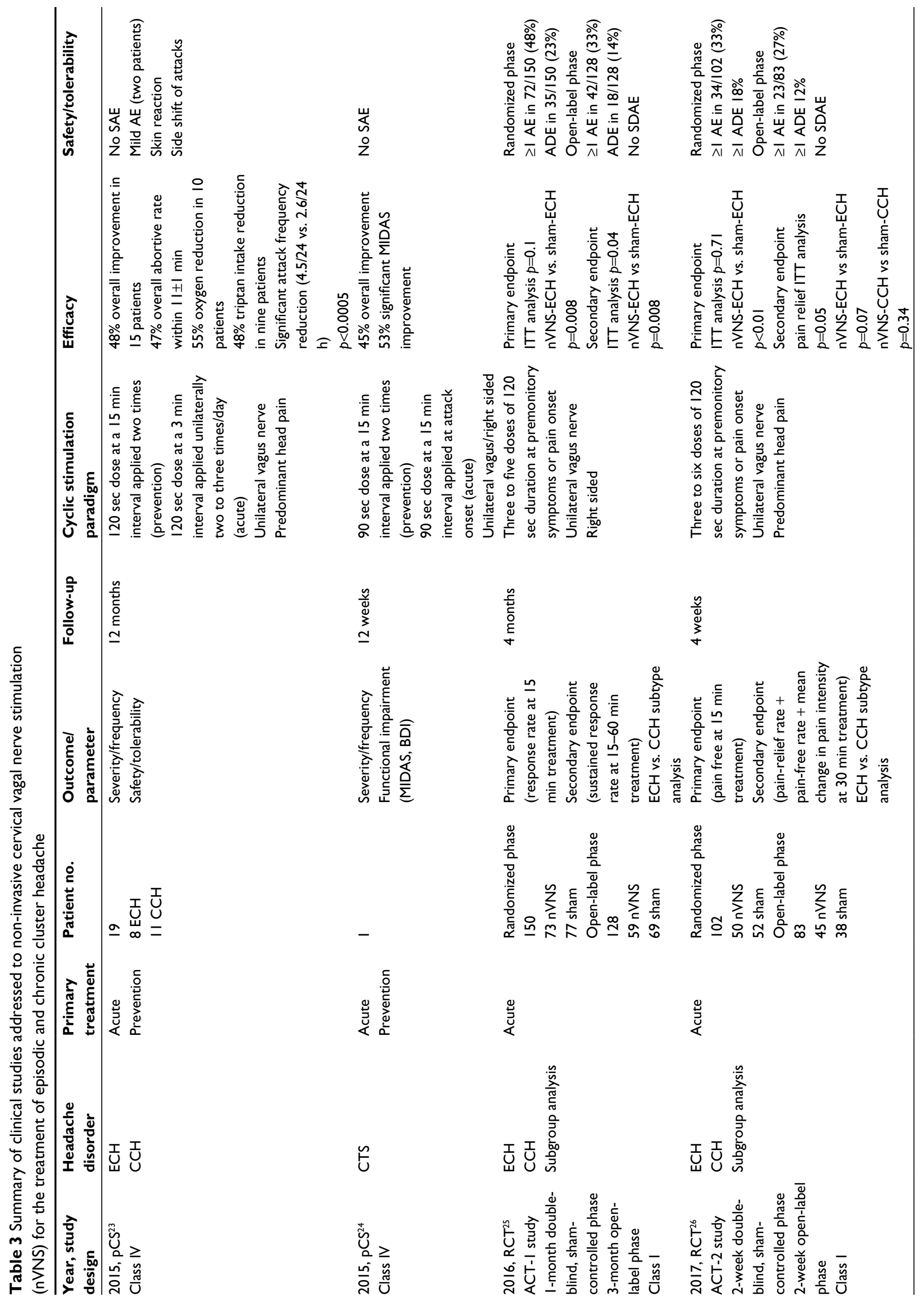




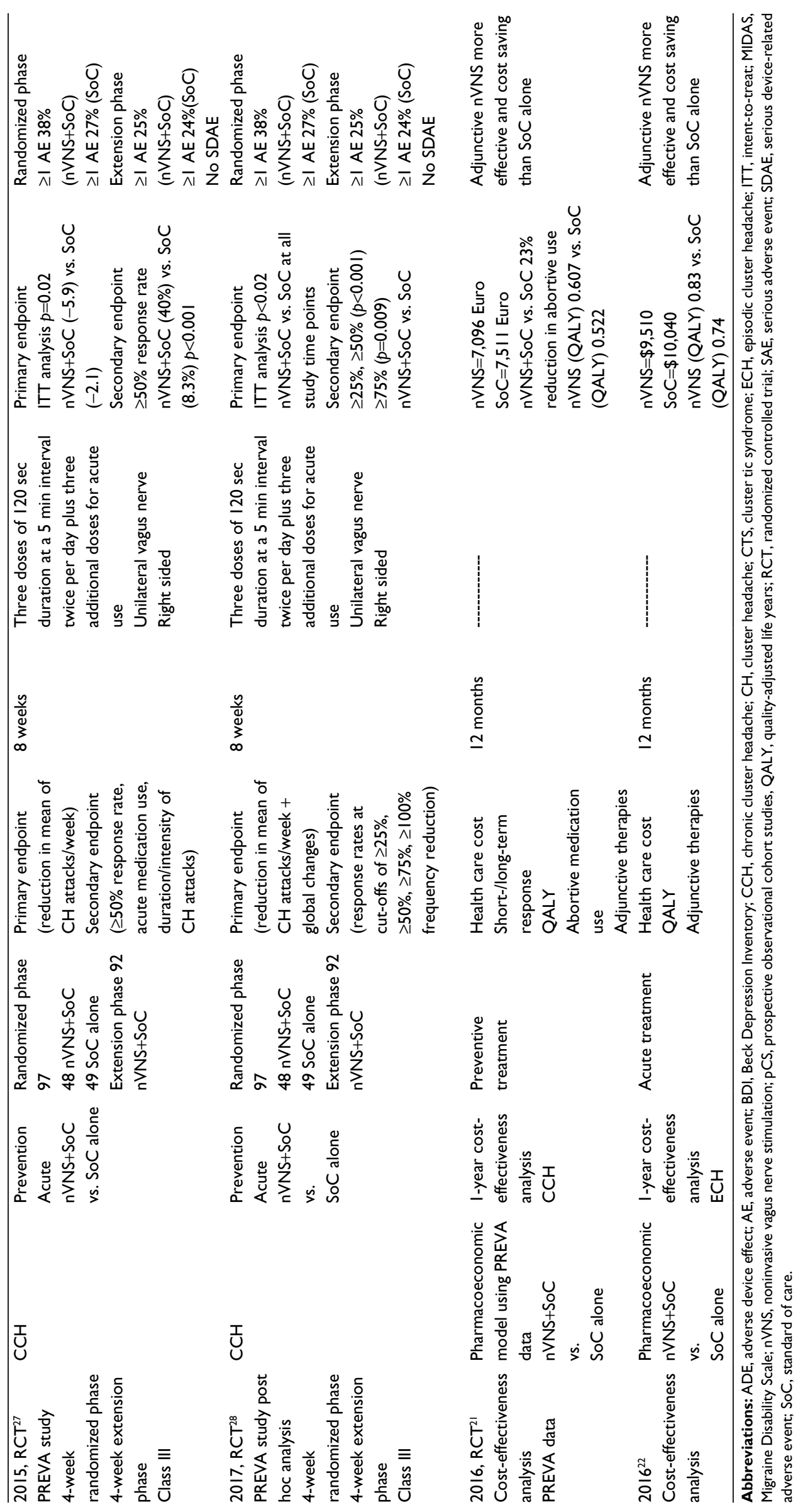


4 weeks adjunctive nVNS (acute protocol: a single $120 \mathrm{sec}$ dose plus additional single $120 \mathrm{sec}$ dose within $1 \mathrm{~h}$ if not pain free, unilateral, right sided). Interestingly, in $47 \%$ of all nVNS-treated attacks, adjunctive rescue medication was not required and $53 \%$ of all attacks required rescue medication. A pain-free state was observed after $1 \mathrm{~h}$ of treatment in $40 \%$ (19/47) and pain relief was observed in 6\% (3/47). ${ }^{16}$ Given these facts, nVNS deserves clinical attention as a considerable and safe alternative in migraine subtypes with impaired therapy options like menstrual-related migraine or migraine in young adolescents.

Although not published, based upon the results of the multicenter, randomized, double-blind, sham-controlled trial, termed PRESTO study (prospective study of nVNS for the acute treatment of migraine), nVNS received the US Food and Drug Administration clearance for abortive therapy in migraine (Class I study). Briefly, nVNS acute treatment resulted in significantly higher rates of pain freedom at 30 , 60 and $120 \mathrm{~min}$ and mean head pain reduction compared to sham stimulation, with a comparable safety/tolerability profile as previously reported. ${ }^{18}$

\section{Episodic and chronic cluster headache}

Nesbitt et al pioneered the prophylactic and acute administration of cervical $\mathrm{nVNS}$ for $\mathrm{ECH}$ and $\mathrm{CCH}$ in a 1-year observational study. ${ }^{23}$ In this initial pilot study, the prophylactic and acute impact of nVNS was observed for $\mathrm{CH}$ (cluster headache) in $19 \mathrm{CH}$ patients (8 ECH and $11 \mathrm{CCH}$; nVNS prevention protocol: $120 \mathrm{sec}$ dose, predominant head pain side, at $3 \mathrm{~min}$ interval 2-3 times/daily $120 \mathrm{sec}$ dose; acute protocol: single $120 \mathrm{sec}$ dose for acute use). A significant decline in attack frequency was observed after 12 months (4.5/24 vs. $2.6 / 24$ h, $p<0.0005)$. Fifteen of 19 participants reported an overall improvement of $48 \%$, and $47 \%$ of all treated attacks were aborted within an average of $11 \pm 1 \mathrm{~min}$. In 10 out of 14 patients (71\%), oxygen demand was decreased by $55 \% \pm 8 \%$, with one patient increasing the oxygen uptake; in nine out of 12 patients (75\%), triptan intake declined by $48 \% \pm 6 \%$, with none increasing the triptan demand. Of note, nVNS was unilaterally administered on the predominant head pain side and encompassed a combined preventive/ acute $n V N S$ protocol.

The ACT-1/ACT-2 studies (Class II studies) represent the first RCT-designed studies for the acute treatment in ECH/ $\mathrm{CCH}$ with adjunctive nVNS with the following stimulation parameters: $120 \mathrm{sec}$ dose, unilateral, predominant head pain side, 3-6 times at premonitory symptoms or at pain onset. ${ }^{25,26}$ ACT-1 and ACT- 2 confirmed the abortive impact of nVNS with a more pronounced responsiveness for $\mathrm{ECH}$ patients. In ACT-1, the randomized phase response rate (defined as the proportion of subjects with pain intensity score of 0 or 1 within $15 \mathrm{~min}$ ) in the total sample was not significantly different ( $p=0.1$ ) compared to sham. However, interestingly, in the ECH subgroup, the response rate was significantly higher after verum stimulation than sham $(p=0.008)$, while there was no significant difference for $\mathrm{CCH}$ patients $(p=0.48)$. In the open-label phase, significantly higher response rates were found in the entire study population $(p=0.04)$ and in the ECH subgroup ( $p=0.008) .{ }^{25}$ The ACT- 2 study confirmed and extended the findings from the ACT-1 study. No significant differences were found in the total sample $(p=0.71)$, while the ECH patients differed significantly (pain-free rate $p<0.01$ ) compared to sham in the randomized 2-weeks phase. In the open-label phase, nVNS was associated with a significantly higher pain-free rate for the total cohort $(p=0.05)$, but not for the $\mathrm{ECH}$ group $(p=0.07)$ and not for the $\mathrm{CCH}$ group $(p=0.34) .{ }^{26} \mathrm{~A}$ pooled assessment of ACT-1 and ACT-2 outcome parameters demonstrated a significantly improved responsiveness for $\mathrm{ECH}$ patients treated with nVNS compared to sham stimulation. ${ }^{27}$

The PREVA study (Class III study) was solely addressed to $\mathrm{CCH}$ patients and compared nVNS plus standard care vs. standard care alone for both prevention and abortive administration. For primary and secondary endpoints, nVNS plus standard care demonstrated significant differences in favor of adjunctive use of nVNS. ${ }^{28}$ The utilized nVNS parameters were as follows: prevention protocol: three doses of $120 \mathrm{sec}$, predominant head pain side, at $5 \mathrm{~min}$ interval, twice/day; acute protocol: $120 \mathrm{sec}$ dose (three times) for acute use. In the randomized phase, the reduction of $\mathrm{CH}$ attacks/week was significantly different between the nVNS+standard of care (SoC) and SoC groups ( -5.9 vs. $-2.1, p=0.02)$ and achieved a $\geq 50 \%$ response rate in the extended phase (nVNS+SoC $40 \%$ vs. Soc $8.3 \%, p<0.001)$. A post hoc analysis of the PREVA data evaluated the mean reduction of $\mathrm{CH}$ attacks/week, global changes (primary endpoint), and as a secondary endpoint, the response rate at cut-offs of $\geq 25 \%, \geq 50 \%$ and $\geq 75 \%$ and $100 \%$ frequency reduction. ${ }^{29}$ At all study time points, $\mathrm{nVNS}+\mathrm{SoC}$ was superior to SoC alone $(p<0.02)$ and the mean weekly attack frequency was significantly decreased within 2 weeks of the randomized phase. nVNS combined with standard care performed significantly better at all study time points and at the response rates defined as cut-offs of $\geq 25 \%, \geq 50 \%$ and $\geq 75 \%$ reduction, with a comparable safety/tolerability outcome between both the groups. ${ }^{28} \mathrm{~A} 1$-year cost-effectiveness analysis was in addition performed on the PREVA data 
$(\mathrm{CCH})$ including the following parameters: health care cost, short-/long-term response, quality-adjusted life years, abortive medication use and additional therapies required. Using these parameters, a pharmacoeconomic model demonstrated lower costs for nVNS+SoC (7096 Euro) vs. SoC alone (7511 Euro), 23\% reduction in abortive medication requirement and a higher quality-adjusted life years index (nVNS+SoC 0.607 vs. SoC 0.522). ${ }^{20,21}$ Mwamburi et al extended the costeffectiveness analysis toward $\mathrm{ECH}$ and the socioeconomic burden of $\mathrm{nVNS}$ vs. SoC alone and found similar results comparable to the PREVA cost-effectiveness assessment. ${ }^{22}$ Table 3 gives a summary of the published literature.

\section{The safety and tolerability profile of cervical nVNS}

The most common AEs of iVNS have been associated with either the surgical implantation procedure (cardiac bradyarrhythmia, infection, bleeding, hardware malfunction) or stimulation-induced complications (cough, voice disturbances, pain) in a considerable proportion of surgical VNS procedures. Contrary to noninvasive VNS, invasive VNS uses a permanent cyclic stimulation paradigm, which may explain in part the higher incidence of stimulation-associated complications observed with iVNS in the past.

According to the current literature, $\mathrm{nVNS}$ has not been associated with serious AEs or serious device-related AEs and the majority of reported AEs remained transient and of mild character (e.g., skin irritation, stiff neck). With respect to the nVNS RCTs (ACT-1/ACT-2, PREVA, EVENT) and the available prospective cohort studies, a low rate of AEs or adverse device-associated events (ADEs) occurred with nVNS treatment in particular, with a similar safety/tolerability profile for nVNS compared to sham stimulation.

\section{MOA of cervical VNS in migraine (human and preclinical studies)}

The neuroinflammatory pathways have been linked to the genesis and maintenance of primary headache disorders such as migraine and cluster headache. Specifically, interactions of interleukins may lead to a disturbed neuroimmune balance.

An increasing body of experimental evidence suggests that VNS modulates the immune response and systemic inflammation by influencing pro- and anti-neuroinflammatory cytokine release (e.g., interleukin [IL]-1 $\beta$, IL-10, IL-6, tumor necrosis factor [TNF]- $\alpha$, HMGB-1, oxytocin) through the cholinergic anti-inflammatory reflex. ${ }^{30-37}$

Perini et al compared the plasma concentrations of proand anti-inflammatory cytokines in migraine patients and
HCs. ${ }^{38}$ Significantly elevated concentrations of intraictal proinflammatory mediators (IL-1 $\beta$, TNF- $\alpha$ ) and antiinflammatory cytokines such as IL-10 were observed compared to postictal values. Interestingly, increased postictal levels declined after acute head pain onset, over time. In healthy subjects, nVNS significantly decreased the plasma levels of proinflammatory IL-1 $\beta$, TNF- $\alpha$, IL-8, MCP-1 and MIP-1 and significantly increased the anti-inflammatory marker IL-10 compared to sham stimulation, indicating that nVNS may downregulate neuroinflammation and thus effectively acts in migraine by restoring the neuroimmune communication. ${ }^{39}$ So far, there exists no human data addressed to possible interactions of $n \mathrm{VNS}$ with the peripheral markers of neuroinflammation in migraine patients.

In order to establish a preclinical model, which parallels the state of recurrent headache attacks or chronic trigeminal nociceptive hypersensitivity, Oshinsky and Gomonchareonsiri exposed dural nociceptors repetitively to an inflammatory infusion. ${ }^{40}$ Quantitative sensory testing (von Frey hair/ monofilaments) of the periorbital region and microdialysis screening in the trigeminal nucleus caudalis (TNC) were implemented in order to assess glyceryl nitrate-evoked changes. After repeated inflammatory infusion, a chronic hypersensitive status along with significantly elevated extracellular glutamate levels was observed. In a later preclinical migraine setting, nVNS (120 sec dose) significantly suppressed extracellular glutamate concentrations without hemodynamic and cardiac side effects. ${ }^{41}$ Serotonin or norepinephrine remained unchanged, indicating that nVNS may be a reasonable treatment approach for trigeminal allodynia.

The impact of preventive migraine drugs (topiramate, valproate, propranolol, amitryptiline) on CSD frequency and the electrical threshold required to initiate CSD propagation was investigated by Ayata et al in another preclinical study using topical application of potassium (applied locally [dura]) or incremental cathodal stimulation. ${ }^{42}$ Chronic administration of prophylactic migraine drugs decreased CSD frequency by $40 \%-80 \%$ and increased cathodal stimulation thresholds for CSD induction. In contrast, acute drug delivery remained without any effect, suggesting CSD analysis to be a suitable approach to determine and develop preventive treatment alternatives. Based on this model, invasive and noninvasive VNS equally suppressed CSD susceptibility in the occipital cortex and increased electrical thresholds by nearly 2-fold either in the ipsilateral or the contralateral hemisphere. Of note, CSD suppression lasted $>3 \mathrm{~h}$ after a $240 \mathrm{sec}$ dose of nVNS application. These observations indicate that $n V N S$ may interact with the development and propagation of CSD as the electrophysiological correlate of migraine aura. ${ }^{43-45}$ 
The genesis of acute migraine pain has been linked with primary afferents activation of the trigeminal nerve/ganglion (TNC) promoted by increased firing of dural nociceptors (vasculature of the dura). The TNC itself projects to the trigemino-cervical complex (TCC), brainstem/medulla oblongata, hypothalamic/thalamic and cortical associated networks (Figure 1). In order to assess the impact of peripheral and central mechanisms in migraine onset, Akerman and Goadsby measured dural vasculature changes and TCC firing response after intravenous and intracerebroventricular administration of the sensory and parasympathetic neuropeptides, vasoactive intestinal peptide (VIP) and pituitary adenylate cyclase-activating peptide 38 (PACAP-38), with receptor-subtype analysis ( $\left.\mathrm{VPAC}_{1 / 2}, \mathrm{PAC}_{1}\right) .^{46}$ Briefly, PACAP-38 and VIP induced dural vessel dilatation via $\mathrm{VPAC}_{2}$-receptor, but VIP was not associated with changes in TCC neuron firing pattern. Neurogenic dural vasodilatation evoked by dural terminals of trigeminal nerve fibers was suppressed by $\mathrm{PAC}_{1}$-receptor antagonist. Intracerebral-ventricular application of PACAP-38, but not of VIP, caused delayed activation and central sensitization of spontaneous TCC firing

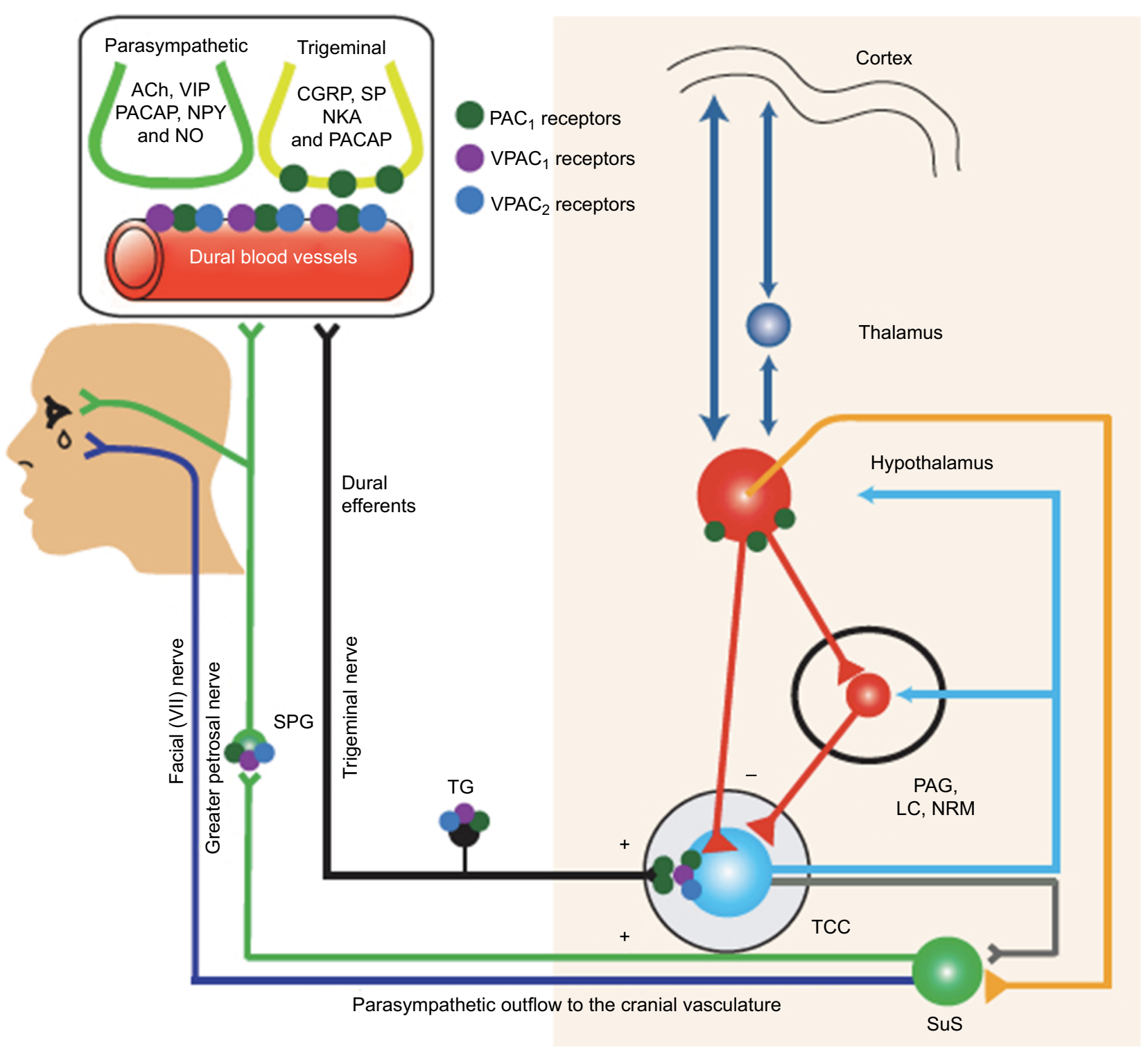

Figure I Schematic drawing of suspected distribution of VPACI/2 and PAC I receptors subtype within the trigeminovascular complex and associated brain circuits. Notes: The parasympathetic neuropeptides vasoactive intestinal peptide (VIP) and pituitary adenylate cylase-activating peptide 38 (PACAP-38) interacts with receptorsubtype analysis (VPACI/2, PAC I) on a central and peripheral level. Neurogenic dural vasodilatation evoked by dural terminals of trigeminal nerve fibers was suppressed via PACI-receptor subdomain. Intra-cerebral-ventricular application of PCAP-38, but not VIP, caused delayed activation and central sensitization of spontaneous TCC ring response (mainly via PACI) along with increased responsiveness to intra- (dural-evoked) and extracranial (cutaneous) stimulation. From Akerman S, Goadsby PJ. Neuronal $\mathrm{PACl}$ receptors mediate delayed activation and sensitization of trigeminocervical neurons: Relevance to migraine. Sci Transl Med. 20I5;7(308):308ral57. Reprinted with permission from AAAS. ${ }^{46}$

Abbreviations: SuS, nucleus salivatorius superior; TG, trigeminal ganglion; TCC, trigeminocervical complex; SPG, ganglion sphenopalatinum; PAG, periaquaeductal grey; LC, locus coeruleus; NRM, nucleus raphe magnus; Ach, acetylcholine; NKA, neurokrinin; CGRP, calcitonin gene-related peptide; SP, substance P. 
response (mainly via $\mathrm{VPAC}_{1}$ and more pronounced $\mathrm{PAC}_{1}$ ) along with increased responsiveness to intracranial (dural-evoked) and extracranial (cutaneous) stimulation. In conclusion, these observations suggest the involvement of endogenous mechanisms in migraine onset rather than dural vascular dilatation. Of note, preclinical nVNS models determined possible interactions with some of these components of the trigemino-nociceptive head pain circuits (Figure 1). ${ }^{46}$

\section{MOA of cervical VNS in cluster headache (human and preclinical data)}

With respect to cluster headache, several human studies determined serum concentrations of pro- and anti-neuroinflammatory cytokines/chemokines compared to HCs. Utilizing a sensitive enzyme-linked immunosorbent assay, a decreased IL-2 receptor expression on the lymphocyte was found in episodic cluster patients. Furthermore, recombinant IL-2 and interferon- $\beta$ were found to counteract this peripheral downregulation. ${ }^{47-49}$ In a later work, Martelletti et al observed that the serum IL- $1 \beta$ level was significantly higher in $\mathrm{ECH}$ patients compared to $\mathrm{HCs}$, intraictally as well as postictally. ${ }^{50}$ In addition, intraictal concentrations were higher than postictal values. IL-1 $\beta$ binds on the hypothalamic receptors, induces corticosteroid secretion and increases substance P synthesis, which itself sensitizes neurons of the autonomic nervous system (sympathetic branch). These multiple reciprocal interactions are believed to be part of a possible feedback loop involved in $\mathrm{CH}$ attack onset and suppression.

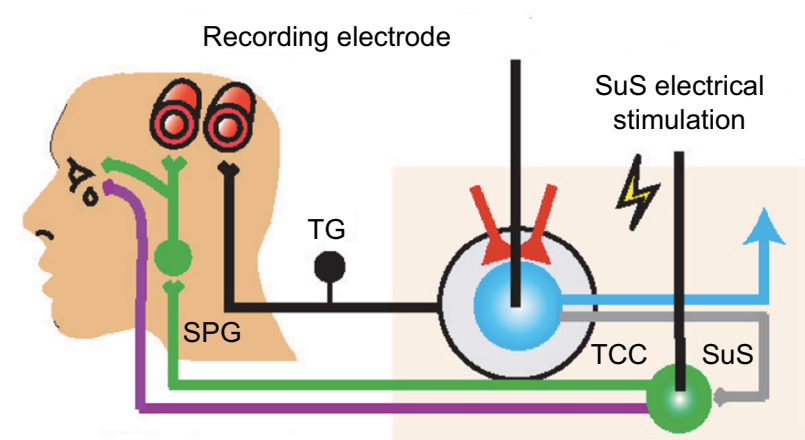

Figure 2 Schematic drawing illustrating the preclinical setting for cluster-like head pain.

Notes: The trigemino-autonomic reflex is suspected to contribute to cluster attack onset and autonomic symptoms (lacrimation flow, nasal congestion). Activation of the nucleus salivatorius superior induces the parasympathetic vasodilatation pathway involving the modulation of TCC neurons and related circuits and evokes autonomic features of cluster headache. From Akerman S, Goadsby PJ. Neuronal $\mathrm{PACl}$ receptors mediate delayed activation and sensitization of trigeminocervical neurons: Relevance to migraine. Sci Transl Med. 2015;7(308):308ra I57. Reprinted with permission from AAAS. ${ }^{46}$

Abbreviations: SuS, nucleus salivatorius superior; TG, trigeminal ganglion; TCC, trigeminocervical complex; SPG, ganglion sphenopalatinum.
In cluster head pain, the trigemino-autonomic reflex is suspected to contribute to cluster attack onset and autonomic symptoms (lacrimation flow, nasal congestion). Activation of the nucleus salivatorius superior induces the parasympathetic vasodilatation pathway involving the modulation of TCC neurons and related circuits (Figure 2). Thus, Akerman and Goadsby developed an acute preclinical approach with the capability to screen migraine-like head pain (dural vascular activation) and cluster-like head pain (trigemino-autonomic reflex). In earlier electrophysiological, preclinical head pain studies, administration of triptans significantly inhibited spontaneous and evoked firing response rates of TCC neurons. ${ }^{51}$

In order to validate the postulated abortive impact of VNS, spontaneous and nociceptive-evoked firing rates of TCC neurons were recorded ipsilaterally and in the contralateral hemisphere. ${ }^{52}$ Dose-dependent changes were observed using a single vs. two $120 \mathrm{sec}$ doses of direct VNS applied at the following parameters: $1 \mathrm{~ms}$ pulse of $5 \mathrm{kHz}$ sine waves repeated at $25 \mathrm{~Hz}$. The VNS dose-response was more pronounced with two $120 \mathrm{sec}$ doses.

After both ipsi- and contralateral stimulation, a dosedependent prolonged decline of spontaneous TCC firing rates was observed after $3 \mathrm{~h}(60 \%)$. Likewise, a suppression of dural-evoked TCC firing by $22 \%$ (A $\alpha$ fiber mediated; fast response) and by $55 \%$ ( $\mathrm{C}$ fiber mediated; slow response) was evident in the VNS-treated group compared to sham stimulation. There were no significant differences between both hemispheres. In the same experimental approach, the nucleus (ncl.) salivatorius superior-induced response (TCC firing rate) was suppressed by $22 \%$ for $2.5 \mathrm{~h}$ after VNS, compared to ipsilateral sham stimulation, and was significantly diminished for ongoing spontaneous TCC neuronal firing. The observed contralateral effects may be indicative of head pain modulation by descending pathways involving the ncl. paraventricularis of the hypothalamus, locus coeruleus and dorsal raphe nucleus. Furthermore, TCC neuron suppression by VNS decreases the likelihood of an attack due to sustained lower TCC thresholds and may explain partly the observed preventive clinical VNS responsiveness. ${ }^{52}$

\section{Conclusion}

Currently published clinical nVNS data demonstrate promising clinical effects for the abortive use in episodic migraine and cluster headache. The interpretation of the findings in this narrative review may be hindered due to several considerations. Although, most of the abortive and preventive trials have been determined as Class I-IV studies (Class I studies for the acute treatment and Class II-IV studies for the preventive use), comparative and reproduc- 
ible conclusions are limited by the different stimulation protocols and/or outcome parameter measures. Secondly, a more systematic review-based approach including multiple comparative correlation analysis of primary and secondary endpoint classified as significantly different or nearlysignificant should re-examine the positive findings of our narrative review. So far, the episodic subtypes of migraine and cluster headache have responded superiorly compared to the chronic forms. Due to its noninvasive character along with the reported tolerability, cervical nVNS may be justified in the pre-refractory state of migraine and cluster headache, and probably in a migraine subpopulation with limited available options (e.g., adolescents with migraine, menstruation-associated migraine). The afferent properties of the vagus nerve are well connected via the ncl. tractus solitarii to the locus coeruleus, the dorsal raphe nucleus, the parabrachial plexus, the paraventricular nucleus of the hypothalamus and directly to the TNC and the cervical spinal cord. Given these anatomic reciprocal projections of the vagus nerve, electrical noninvasive modulation of the cervical vagal afferents may impact trigeminovascular nociceptive transmission.

Along with a rising number of targeted preclinical studies supporting the observed clinical VNS responsiveness in primary headache disorders, the authors strongly believe that VNS constitutes an emerging issue of ongoing headache treatment and research.

\section{Acknowledgment}

The authors want to express their thanks to Bruce J Simon (PhD) for giving important intellectual input.

\section{Disclosure}

Thomas M Kinfe has received training support, and works as a consultant for St Jude Medical, Inc. and Medtronic Inc. The authors report no other conflicts of interest in this work.

\section{References}

1. Howland RH. Vagus nerve stimulation. Curr Behav Neurosci Rep. 2014;1(2):64-73.

2. Beekwilder JP, Beems T. Overview of the clinical applications of vagus nerve stimulation. J Clin Neurophysiol. 2010;27(2):130-138.

3. Ben-Menachem E, Revesz D, Simon BJ, Silberstein S. Surgically implanted and non-invasive vagus nerve stimulation: a review of efficacy, safety and tolerability. Eur J Neurol. 2015;22(9):1260-1268.

4. The International Classification of Headache Disorders, 3rd edition (beta version). Cephalalgia. 2013;33(9):629-808.

5. Lenaerts ME, Oommen KJ, Couch JR, Skaggs V. Can vagus nerve stimulation help migraine? Cephalalgia. 2008;28(4):392-395.

6. Sadler RM, Purdy RA, Rahey S. Vagal nerve stimulation aborts migraine in patient with intractable epilepsy. Cephalalgia. 2002;22: $482-484$.
7. Hord ED, Evans MS, Mueed S, Adamolekun B, Naritoku DK. The effect of vagal nerve stimulation on migraine. J Pain. 2003;4:530-534.

8. Mauskop A. Vagus nerve stimulation relieves chronic refractory migraine and cluster headaches. Cephalalgia. 2005;25:82-86.

9. Cecchini AP, Mea E, Tullo V, et al. Vagus nerve stimulation in drugresistant daily chronic migraine with depression: preliminary data. Neurol Sci. 2009;30(1):101-104.

10. Pintea B, Hampel K, Boström J, et al. Extended long-term effects of cervical vagal nerve stimulation on headache intensity/frequency and affective/cognitive headache perception in drug resistant complexpartial seizure patients. Neuromodulation. 2017;20(4):375-382.

11. Goadsby, P. J., Grosberg BM, Mauskop A, Cady R, Simmons KA. Effect of noninvasive vagus nerve stimulation on acute migraine: an open-label pilot study. Cephalalgia. 2014;34(12):986-993.

12. Barbanti P, Grazzi L, Egeo G, Padovan AM, Liebler E, Bussone G. Noninvasive vagus nerve stimulation for acute treatment of high-frequency and chronic migraine: an open-label study. J Headache Pain. 2015;16:61.

13. Kinfe TM, Pintea B, Muhammad S, et al. Cervical non-invasive vagus nerve stimulation (nVNS) for preventive and acute treatment of episodic and chronic migraine and migraine-associated sleep disturbance: a prospective observational cohort study. J Headache Pain. 2015;16:101-107.

14. Silberstein SD, Calhoun AH, Lipton RB, et al. Chronic migraine headache prevention with non-invasive vagus nerve stimulation: the EVENT study. Neurology. 2016;87:1-10.

15. Grazzi L, Egeo G, Calhoun AH, McClure CK, Liebler E, Barbanti P. Non-invasive Vagus Nerve Stimulation (nVNS) as mini-prophylaxis for menstrual/menstrually related migraine: an open-label study. $J$ Headache Pain. 2016;17(1):91.

16. Grazzi L, Egeo G, Liebler E, et al. Non-invasive Vagus Nerve Stimulation (nVNS) as symptomatic treatment for migraine in young patients: a preliminary safety study. Neurol Sci. 2017;38(Suppl 1):S197-S199.

17. electroCore. News. Electrocore receives FDA approval for chronic migraine study. Available from: http:/www.electrocore.com/electrocore-receives-fda-approval-for-chronic-migraine-study. Accessed September 14, 2014.

18. electroCore. News. Electrocore receives FDA clearance for acute treatment of pain associated with migraine headache in adult patients. Available from http://www.electrocore.com/3518-2. Accessed January 29, 2018.

19. Holle-Lee D, Gaul C. Noninvasive vagus nerve stimulation in the management of cluster headache: clinical evidence and practical experience. Ther Adv Neurol Disord. 2016;9(3):230-234.

20. Jenks M, Davis S, Amato F, Errico J, Strickland I. A preliminary cost-utility analysis of non-invasive vagusnerve stimulation therapy in patients suffering with headache and functional disorder multi-morbidity. ISPOR 2016: 19th Annual European Congress of the International Society for Pharmacoeconomics and Outcomes Research (ISPOR); Health Economics Consortium; 2016 Oct 29 - Nov 2, Vienna, Austria.

21. Morris J, Straube A, Diener HC, et al. Cost-effectiveness analysis of non-invasive vagus nerve stimulation for the treatment of chronic cluster headache. J Headache Pain. 2016;17:43.

22. Mwamburi M, Liebler E, Tenaglia AT. Cost-effectiveness of gammaCore (non-invasive vagus nerve stimulation) for acute treatment of episodic cluster headache. Am J Manag Care. 2017;23(16 Suppl):S300-S306.

23. Nesbitt AD, Marin JC, Tompkins E, Ruttledge MH, Goadsby PJ. Initial use of a novel noninvasive vagus nerve stimulator for cluster headache treatment. Neurology. 2015;84(12):1249-1253.

24. Kinfe TM, Pintea B, Güresir E, Vatter H. Partial response of intractable cluster-tic syndrome treated by cervical non-invasive vagal nerve stimulation (nVNS). Brain Stimul. 2015;8(3):669-671.

25. Silberstein SD, Mechtler LL, Kudrow DB, et al. Non-invasive vagus nerve stimulation for the acute treatment of cluster headache: findings from the randomized, double-blind, sham-controlled ACT1 study. Headache. 2016;56(8):1317-1332.

26. Goadsby PJ, de Coo IF, Silver N, et al. ACT2 Study Group. Non-invasive vagus nerve stimulation for the acute treatment of episodic and chronic cluster headache: a randomized, double-blind, sham-controlled ACT2 study. Cephalalgia. 2018;38(5):959-969. 
27. Coo I, Marin JCA, Silberstein SD, et al. Non-invasive Vagus Nerve Stimulation (nVNS): Acute Treatment of Episodic and Chronic Cluster Headache: Pooled Analysis of ACT1 and ACT2 Studies. Paper presented at: AAN2017; Boston.

28. Gaul C, Diener HC, Silver N, et al. Non-invasive vagus nerve stimulation for PREVention and Acute treatment of chronic cluster headache (PREVA): a randomized controlled study. Cephalalgia. 2015;36(6):534-546.

29. Gaul C, Magis D, Liebler E, Straube A. Effects of non-invasive vagus nerve stimulation on attack frequency over time and expanded response rates in patients with chronic cluster headache: a post-hoc analysis of the randomized, controlled PREVA study. J Headache Pain. 2017;18(1):22.

30. Tracey KJ. Physiology and immunology of the cholinergic anti-inflammatory pathway. J Clin Invest. 2007;117(2):289-296.

31. Kemper RH, Meijler WJ, Korf J, Ter Horst GJ. Migraine and function of the immune system: a meta-analysis of clinical literature published between 1966 and 1999. Cephalalgia. 2001;21:549-557.

32. Pavlov VA, Tracey KJ. The cholinergic anti-inflammatory pathway. Brain Behav Immun. 2005;19(6):493-499.

33. Pavlov VA, Tracey KJ. The vagus nerve and the inflammatory reflex linking immunity and metabolism. Nat Rev Endocrinol. 2012;8:743-754.

34. Hasko G, Szabo C. Regulation of cytokine and chemokine production by transmitters and co-transmitters of the autonomic nervous system. Biochem Pharmacol. 1998;56:1079-1087.

35. Vezzani A, Viviani B. Neuromodulatory properties of inflammatory cytokines and their impact on neural excitability. Neuropharmacology. 2015;96:70-82.

36. Wang $\mathrm{H}, \mathrm{Yu} \mathrm{M}$, Ochani $\mathrm{M}$ et al. Nicotinic acetylcholine receptor alpha7 subunit is an essential regulator of inflammation. Nature. 2003;421 384-388.

37. Vida, G., Peña, G., Deitch, Ulloa U. $\alpha 7$ - cholinergic receptor mediates vagal induction of splenic norepinephrine. J Immunol. 2011;186(7):4340-4346.

38. Perini F, D`Andrea G, Galloni E, et al. Plasma cytokine levels in migraineurs and controls. Headache. 2005;45:926-931.

39. Lerman I, Hauger R, Sorkin L, et al. Noninvasive transcutaneous vagus nerve stimulation decreases whole blood culture-derived cytokines and chemokines: a randomized, blinded, healthy control pilot trial. Neuromodulation. 2016;19(3):283-290.
40. Oshinsky ML, Gomonchareonsiri S. Episodic dural stimulation in awake rats: a model for recurrent headache. Headache. 2007;47(7):1026-1036.

41. Oshinsky ML, Murphy AL, Hekierski H, Cooper M, Simon BJ. Noninvasive vagus nerve stimulation as treatment for trigeminal allodynia. Pain. 2014;155:1037-1042.

42. Ayata C, Jin H, Kudo C, Dalkara T, Moskowitz MA. Suppression of cortical depression in migraine prophylaxis. Ann Neurol. 2006;59:652-661.

43. Chen SP, Ay I, de Morais AL, et al. Vagus nerve stimulation inhibits cortical spreading depression susceptibility. Pain. 2016;157(4): 797-805.

44. Chen SP, Ayata C. Spreading depression in primary and secondary headache disorders. Curr Pain Headache Rep. 2016;20(7):44.

45. Karatas H, Erdender SE, Gursoy-Ozdemir Y et al. Spreading depression triggers headache by activating neuronal Panx1 channels. Science. 2013;339:1092-1095.

46. Akerman S, Goadsby PJ. Neuronal PAC1 receptors mediate delayed activation and sensitization of trigeminocervical neurons: relevance to migraine. Sci Transl Med. 2015;7(308):308ra157.

47. Martelletti P, Stirparo G, De Stefano L, Di Sabato F, Giacovazzo M, Rinaldi-Garaci C. Defective expression of IL-2 receptors on peripheral blood lymphocytes from patients with cluster headache. Headache. 1990;30:228-231

48. Stirparo G, Martelletti P, Morrone S, Savarese, A, Giacovazzo, M. Impaired natural killer activity in PBLs from cluster headache patients is restored by interleukin 2. Int J Immunother. 1990;6:181-186.

49. Martelletti P, Stirparo G, De Stefano L, Rinaldi-Garaci, C, Giacovazzo, M. Studies on lymphokine-activated killer cells and natural killer cells in cluster headache: increased sensitivity to RIL-2 and IFN-B. In: Rose C, editors. New Advances in Headache Research. London: Smith Gordon; 1989:139-144.

50. Martelletti P, Granata M, Giacovazzo M. Serum interleukin-1 beta is increased in cluster headache. Cephalalgia. 1993;13(5):343-350.

51. Akerman S, Goadsby PJ. A novel translational animal model of trigeminal autonomic cephalalgias. Headache. 2015;55(1):197-203.

52. Akerman S, Simon B, Romero-Reyes M. Vagus nerve stimulation suppresses acute noxious activation of trigeminiocervical neurons in animal models of primary headache. Neurobiol Dis. 2017;102: 96-104.

\section{Journal of Pain Research}

\section{Publish your work in this journal}

The Journal of Pain Research is an international, peer reviewed, open access, online journal that welcomes laboratory and clinical findings in the fields of pain research and the prevention and management of pain. Original research, reviews, symposium reports, hypothesis formation and commentaries are all considered for publication.

\section{Dovepress}

The manuscript management system is completely online and includes a very quick and fair peer-review system, which is all easy to use. Visit http://www.dovepress.com/testimonials.php to read real quotes from published authors. 